\title{
Influence de l'origine géographique du lait sur la précision des dosages de matière grasse et de protéines par spectroscopie dans le moyen infrarouge
}

\author{
O. Leray *
}

INRA, Station de recherches de technologie et analyses laitières 39800 Poligny, France

(reçu le 14 décembre 1988, accepté le 17 juillet 1989)

Résumé - Trois essais menés sur trois périodes différentes de production laitière ont permis d'évaluer l'influence de la région sur la précision des dosages par spectrophotométrie dans le moyen infrarouge de la matière grasse, à $5,73 \mu \mathrm{m}$ (filtre A) et à $3,48 \mu \mathrm{m}$ (flitre B), et des protéines à $6,46 \mu \mathrm{m}$. Cette influence s'est avérée importante en matière grasse à $5,73 \mu \mathrm{m}$, faible en matière grasse à $3,48 \mu \mathrm{m}$ et quasi inexistante pour les dosages de protéines. Un système d'étalonnage unique centralisé pour l'ensemble des régions pourrait être envisagé sans restriction pour ces deux derniers types d'analyses. Pour les dosages à $5,73 \mu \mathrm{m}$, une procédure d'ajustement local devrait accompagner l'étalonnage à distance.

analyse du lait - moyen infrarouge — influence régionale — étalonnage centralisé

Summary - Effect of geographical origin on the accuracy of milk fat and protein measurements by mid infra-red spectroscopy. Three experiments were carried out at three different milk production periods. The influence of a different area of milk production on the mid infra-red fat test at $5.73 \mu \mathrm{m}$ ( $A$ filter) and at $3.48 \mu \mathrm{m}$ (B filter), and on the mid infra-red true protein test at $6.46 \mu \mathrm{m}$ was measured. The regional effect was important on $5.73 \mu \mathrm{m}$ fat determinations, rather weak at $3.48 \mu \mathrm{m}$ and almost non significant on protein analyses. Only a centralized system of calibration of I.R. instruments can pe envisaged for the two latter kinds of analysis. Concerning analysis at $5.73 \mu \mathrm{m}$, local fittings shoud be required in addition to centralized calibration.

milk — mid infra-red analysis — regional effect - centralized calibration

\footnotetext{
* Ingénieur ITEB (Institut Technique de l'Elevage Bovin).
} 


\section{INTRODUCTION}

L'analyse des principaux constituants du lait, matière grasse et protéines (accessoirement lactose), par spectrophotométrie dans le moyen infrarouge s'est généralisée au cours de ces 15 dernières années dans les laboratoires d'analyse de routine travaillant pour le contrôle laitier individuel ou pour le paiement du lait.

Les premiers appareils mis en place dans les années soixante-dix, progressivement modifiés et améliorés tout en conservant les mêmes principes de base, ont atteint un niveau de performance et de fiabilité répondant aux attentes des utilisateurs.

Néammoins l'amélioration des performances instrumentales, si elle a apporté un gain de fidélité (répétabilité et stabilité de la réponse des appareils), n'a que peu modifié les problèmes relatifs à l'étalonnage des appareils, essentiellement liés à la composition physicochimique des constituants du lait.

On sait notamment que les dosages de matière grasse à $5,73 \mu \mathrm{m}$ (dosage avec filtre A) sont fortement influencés par le poids moléculaire moyen des molécules de triglycérides, lui-même lié à la longueur des chaînes des acides gras constitutifs (Shields, 1975). En effet, le rayonnement est absorbé au niveau des liaisons esters des glycérides et ne tient pas compte des chaînes carbonées qui constituent l'essentiel du volume ou du poids de la matière grasse.

Ainsi tout facteur susceptible de faire varier la composition moyenne de la matière grasse est un facteur potentiel d'imprécision : alimentation liée au régime, à la saison ou à la région, race (Sjaunja, 1982), espèce (Brochet 1982; Grappin \& Jeunet, 1979), stade de lactation (Grappin \& Jeunet, 1972; Leray, 1984; Sjaunja, 1982).
L'utilisation d'une autre longueur d'onde, $3,48 \mu \mathrm{m}$ (filtre B), absorbée au niveau des liaisons carbone-hydrogène réduit l'influence qualitative de la matière grasse en prenant en compte la dimension des chaînes des acides gras. D'une manière générale, la précision des dosages s'en trouve accrue (Kerkhof et al., 1982), bien que l'on note une sensibilité particulière de ce type de dosage au degré d'insaturation des chaînes d'acide gras.

Les protéines sont dosées à $6,46 \mu \mathrm{m}$ par absorption du rayonnement au niveau des liaisons peptidiques. A cette longueur d'onde absorbent également les fonctions acides carboxyliques sous forme dissociée (influence des citrates naturels [Gaudillère \& Grappin, 1982; Sjaunja \& Anderson, 1985], de l'acidification lactique [Sjaunja, 1982], d'acides gras libre provenant d'un lipolyse [Grappin \& Jeunet, 1981; Sjaunja, 1982]).

Le lactose est également un composant qui interfère à 6,46 $\mu \mathrm{m}$ (Grappin \& Jeunet, 1976), mais il est possible de compenser son influence par application d'une correction proportionnelle au taux de lactose qui doit être dosé parallèlement vers $9,6 \mu \mathrm{m}$.

Grappin \& Jeunet (1972) ont montré l'importance de l'évolution saisonnière des étalonnages dans les dosages de matière grasse à $5,73 \mu \mathrm{m}$, en relation avec l'évolution de l'indice de réfraction utilisé comme indicateur du poids moléculaire moyen de la matière grasse.

Une étude menée sur les trois espèces bovine, ovine et caprine, par Brochet (1982) témoigne d'une relative stabilité saisonnière des étalonnages en ce qui concerne la matière grasse à $3,48 \mu \mathrm{m}$ et les protéines, alors qu'elle observe d'importantes variations en matière grasse à $5,73 \mu \mathrm{m}$.

Etudiant par ailleurs la variabilité régionale des étalonnages, Brochet (1982) a 
montré des différences allant jusqu'à $1 \mathrm{~g} / \mathrm{l}$ sur des laits de brebis provenant d'Aveyron, du rayon de Roquefort, des Pyrénnées Atlantiques et de Corse.

Dans la pratique, la diversité des matériels utilisés et les causes naturelles d'imprécision (diversité notamment des systèmes alimentaires variables avec la saison) ont imposé sur le groupe de 43 laboratoires interprofessionnels et de contrôle laitier actuels, l'adoption d'un système d'étalonnage individuel, laboratoire par laboratoire, conformément à une norme d'utilisation des appareils infrarouge (ITEB-FNOCL, 1983).

Ce système est essentiellement dicté par une forte proportion d'appareils munis de filtres $A(5,73 \mu \mathrm{m})$ dans le parc des instruments des laboratoires français. Dans le cas de l'utilisation du filtre $B(3,48 \mu \mathrm{m})$ moins sensible aux phénomènes de composition, il n'est pas exclu que les effets saisonniers et régionaux puissent être négligés. De ce fait, une large diffusion de ce type de dosage pourrait rendre caduc le principe d'étalonnage individuel au profit d'un étalonnage unique centralisé.

L'objectif de ce travail est essentiellement d'apprécier l'influence de l'origine des échantillons sur la justesse des mesures infrarouge afin d'évaluer la possibilité d'utiliser des échantillons standards préparés à partir d'un laboratoire central pour le dosage de la matière grasse à $5,73 \mu \mathrm{m}$ et à $3,48 \mu \mathrm{m}$ et celui des protéines.

Les essais ont donc consisté à mesurer, pour chaque longueur d'onde, avec un même appareil, les écarts de résultats instrumentaux obtenus sur des échantillons de lait de provenances diverses, aux résultats obtenus avec la méthode de référence correspondante. Le regroupement des écarts par région et la comparaison des différentes régions entre elles sur la base des moyennes observées ont permis d'évaluer le degré de proximité des différentes régions dans le cas d'un étalonnage commun.

\section{MATÉRIEL ET MÉTHODES}

Trois essais indépendants ont été réalisés au cours de 3 campagnes laitières, mais concernant des époques de production différentes de manières à apprécier en partie l'effet saison :

- essai 1 : période de changement alimentaire, novembre 1981;

- essai 2 : période d'alimentation d'hiver, février 1984;

- essai 3 : période d'alimentation d'été, juin 1985.

\section{Nature des prélèvements}

Lors des 3 essais, les prélèvements ont été effectués par les agents nationaux du contrôle laitier (ANCL) de PITEB sur une période de 1 semaine.

Respectivement, 102,115 et 79 échantillons de lait de tank (de 2 à 4 traites) ont été prélevés dans 6 à 8 zones ou "régions". Chaque région, issue d'un découpage arbitraire mais dotée d'un ou plusieurs laboratoires, est composée de 1 à 3 départements, parties du secteur d'activité d'un agent, et est représentée par 8 à 15 échantillons.

La Figure 1 permet de visualiser la dispersion géographique des diverses zones de prélèvement, la liste des départements et des zones étant reportée au Tableau I.

Les troupeaux ont été choisis pour représenter au mieux les élevages locaux et présenter une étendue de taux aussi large que possible.

Les vaches productrices de ces laits étaient pour $70 \%$ de race FFPN et FFxHolstein localisées dans l'ouest, le nord et le nord-est, pour $20 \%$ de race Montbéliarde essentiellement localisées dans l'est, et pour $10 \%$ de race Nor- 
mande situées en Calvados, Deux-Sèvres et Mayenne.

Dès le prélèvement, les échantillons ont été munis d'un conservateur (bronopol à $0,02 \%$ dans le lait) et acheminés soit par la route, soit par la poste, dans les $72 \mathrm{~h}$ à la station expérimentale laitière de Poligny pour y être analysés une fois tous réunis.

Dans les Tableaux II à V sont reportées, entre autres résultats, les plages de variation des taux rencontrés (minima, maxima et écarts types).

\section{Analyses}

Les échantillons ont été analysés dans un ordre tel qu'aucun biais analytique ne puisse accroître artificiellement un effet régional.

\section{Analyses de référence}

Les méthodes utilisées dans les laboratoires de routine ont été employées :

- matière grasse : méthode Gerber (norme AFNOR V 04-210);
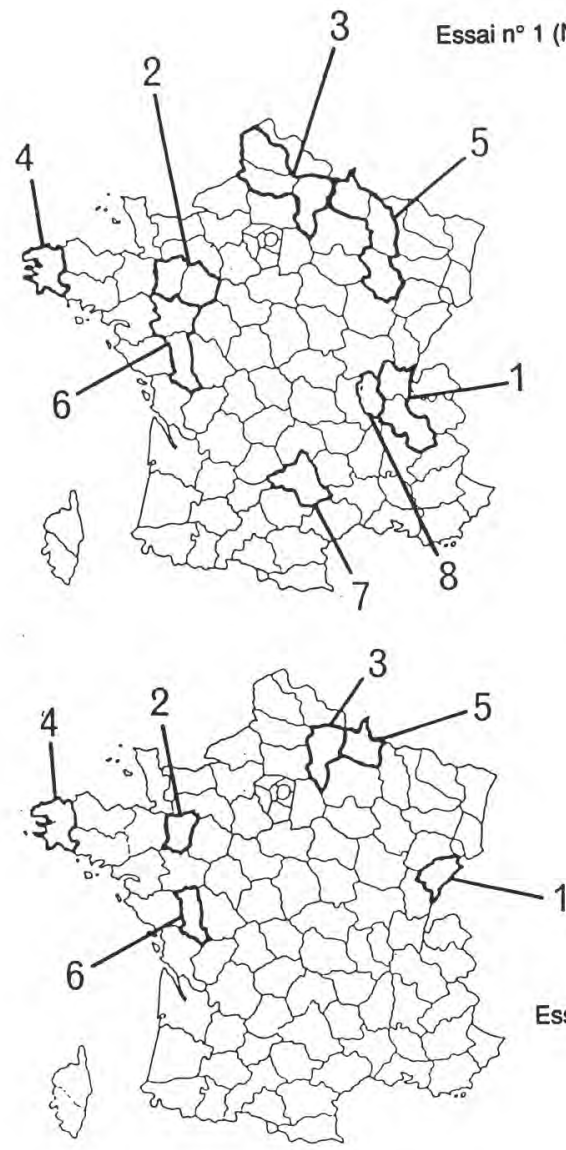

Essai nº 2 (Février 1984)

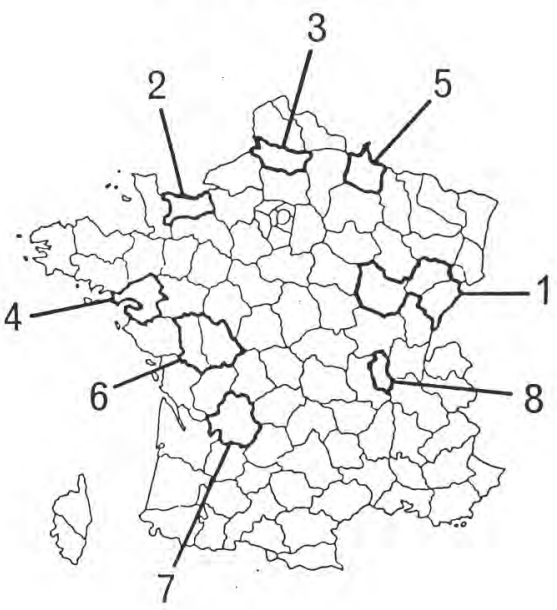

Essai $n^{\circ} 3$ (Juin 1985)

Fig. 1. Répartition des zones de prélèvement ou «régions" des 3 essais d'appréciation de l'influence régionale sur la précision des dosages de matière grasse et de protéines en moyen infrarouge et sur les calibrages. 
Tableau I. Répartition des zones de prélèvement au cours des différents essais.

\section{Régions}

A : novembre 1981

$\begin{array}{llr}1 & \text { Isère (38) et Ain (01) } & 15 \\ 2 & \text { Sarthe (72) et Mayenne (53) } & 8 \\ 3 & \text { Pas-de-Calais (62), Somme (80) et Aisne (02) } & 15 \\ 4 & \text { Finsitère (29) } & 8 \\ 5 & \text { Haute-Marne (52), Meuse (55) et Ardennes (08) } & 11 \\ 6 & \text { Maine-et-Loir (49) et Deux-Sèvres (79) } & 15 \\ 7 & \text { Aveyron (12) } & 15 \\ 8 & \text { Rhône (Monts du Lyonnais - 69) } & 15\end{array}$

B : février 1984

1

2

3

4

5

6

7

8

C : juin 1985
Côte d'Or (21), Doubs (25) et Haute-Saône (70) 15

Calvados (14)

Somme (80)

Loire-Atlantique (44)

Ardennes (08)

Deux-Sèvres (79) et Vienne (86)

Dordogne (24)

Rhône (Monts du Lyonnais - 69)
15

8

15

8

11

15

15

Doubs (25) $\quad 15$

Mayenne (53) 7

Aisnes (02) $\quad 15$

Finistère (29) 13

Ardennes (08) 14

Deux-Sèvres (79) 15

- protéines : méthode au Noir Amido, préalablement étalonnée par rapport à la méthode Kjeldahl à l'aide d'échantillons de référence, pour les protéines ((MAT-ANP) x 6,38).

Les analyses Gerber ont été effectuées en simple mais ont été confirmées par un double dans le cas d'un résidu référence-infrarouge supérieurs à $1,5 \mathrm{~g} / \mathrm{kg}$; les analyses Noir Amido ont été faites en double systématiquement, les moyennes des doubles ayant servi de référence.

\section{Analyses infrarouge}

L'appareil utilisé a été un MilkoScan 104 (FossElectric, Danemark) permettant le dosage de la matière grasse à $5,73 \mu \mathrm{m}$ (filtre A) et à $3,48 \mu \mathrm{m}$ (filtre B), des protéines à $6,46 \mu \mathrm{m}$ et du lactose à $9,6 \mu \mathrm{m}$.

Les échantillons ont été analysés en double (deux passages successifs de même série), de manière à en exploiter les moyennes. 
Les analyses infrarouge ont été réalisées en deux passages successifs sur l'appareil, correspondant à deux sélections différentes des canaux d'analyse :

- sélection des quatre longueurs d'onde disponibles qui ont fourni les résultats de matière grasse, en filtre $A$ et en filtre $B$, et de protéines corrigés de l'influence du taux de lactose;

- sélection des deux filtres matière grasse $A$ et protéines, de manière à obtenir les résultats protéines en l'absence de l'intercorrection lactose.

\section{Traitement des résultats}

Chaque essai a été traité individuellement selon le même schéma, pour chaque type de dosage :

- calcul de régression linéaire selon la méthode des moindres carrés sur l'ensemble des couples (référence-appareil), les valeurs de référence étant expliquées par les valeurs instrumentales;

- calcul des résidus des régressions, puis analyses de variance à un facteur sur les résidus avec la région comme source de variation;

- comparaison 2 à 2 des moyennes de résidus par région.

\section{RÉSULTATS}

\section{Les dosages de matière grasse}

Filtre A $(5,73 \mu \mathrm{m})$

Les écarts types résiduels des régressions, observés respectivement pour les essais sont de $0,82,0,43$ et $0,51 \mathrm{~g} / \mathrm{kg}$ ( $\mathrm{Ta}$ bleau II).

\section{Premier essai}

L'analyse de variance et les comparaisons par paires montrent une influence significative au seuil de $1 \%$ de l'effet région. La région Aveyron est principalement cause de cet effet hautement significatif avec un écart moyen à la régression de $-0,69 \mathrm{~g} / \mathrm{kg}$ (Tableau II).

L'amplitude maximale observée entre les moyennes régionales est de $1,02 \mathrm{~g} / \mathrm{kg}$. En/l'absence de la région Aveyron (région 7), elle est réduite à $0,62 \mathrm{~g} / \mathrm{kg}$. L'influence régionale n'apparaît alors plus significative $(F=1,313)$, masquée par l'importante variabilité des résidus intrarégion. Les écarts types régionaux varient de 0,43 à $1,07 \mathrm{~g} /$ $\mathrm{kg}$, avec une moyenne de $0,77 \mathrm{~g} / \mathrm{kg}$.

On peut noter que les régions d'influence océanique forment un groupe très homogène avec des moyennes de résidus comprises entre + 0,28 et $0,33 \mathrm{~g} / \mathrm{kg}$ (régions 2, 3, 4 et 6 ). Elles s'opposent dans la régression linéaire aux régions plus continentales qui apparaissent nettement moins cohérentes entre elles.

\section{Deuxième essai}

II ne présente pas d'influence régionale significative. On peut isoler deux groupes homogènes de régions non distinctes, qui ne se différencient que par les zones extrêmes 2 et 7 (Calvados et Dordogne). L'amplitude des écarts moyens à la régression est de 0,42 g/kg.

Les écarts types des résidus par région fluctuent entre 0,28 et $0,59 \mathrm{~g} / \mathrm{kg}$ avec une moyenne de $0,43 \mathrm{~g} / \mathrm{kg}$.

\section{Troisième essai}

II montre un effet région très hautement significatif $(P<0,05 \%)$, provenant essentiel- 
Tableau II. Tableau des moyennes (d) et des écarts types régionaux (Sd) des écarts aux régressions linéaires observés en dosage de matière grasse à $5,73 \mu \mathrm{m}$, sur les 3 essais successifs.

\begin{tabular}{|c|c|c|c|c|c|c|c|c|c|c|c|c|c|c|c|}
\hline \multirow{3}{*}{$\begin{array}{l}\text { région } \\
1\end{array}$} & \multicolumn{5}{|c|}{ Essai 1 : novembre 1981} & \multicolumn{5}{|c|}{ Essai 2 : février 1984} & \multicolumn{5}{|c|}{ Essai 3 : juin 1985} \\
\hline & \multirow{2}{*}{$\begin{array}{c}n \\
15\end{array}$} & \multirow{2}{*}{$\begin{array}{c}\text { min. } \\
\text { max. } \\
30,7 \\
43,0\end{array}$} & \multirow{2}{*}{$\begin{array}{r}d \\
+0,07\end{array}$} & \multicolumn{2}{|l|}{$S d$} & \multirow{2}{*}{$\begin{array}{c}n \\
15\end{array}$} & \multirow{2}{*}{$\begin{array}{c}\text { min. } \\
\text { max. } \\
33,0 \\
44,0\end{array}$} & \multirow{2}{*}{$\begin{array}{c}d \\
-0,04\end{array}$} & \multicolumn{2}{|l|}{$S d$} & \multirow{2}{*}{$\begin{array}{c}n \\
15\end{array}$} & \multirow{2}{*}{$\begin{array}{c}\text { min. } \\
\max .\end{array}$} & \multirow{2}{*}{$\begin{array}{c}d \\
-0,28\end{array}$} & \multicolumn{2}{|c|}{$S d$} \\
\hline & & & & 0,43 & $a b$ & & & & 0,39 & $a b$ & & & & 0,46 & a \\
\hline 2 & 8 & $\begin{array}{l}39,3 \\
49,0\end{array}$ & $+0,28$ & 0,72 & $a b$ & 15 & $\begin{array}{l}33,0 \\
44,1\end{array}$ & $+0,22$ & 0,47 & b & 6 & $\begin{array}{l}34,0 \\
40,4\end{array}$ & $-0,22$ & 0,56 & $a b$ \\
\hline 3 & 15 & $\begin{array}{l}34,3 \\
50,6\end{array}$ & $+0,33$ & 0,78 & b & 15 & $\begin{array}{l}35,9 \\
46,9\end{array}$ & $+0,02$ & 0,38 & $a b$ & 15 & $\begin{array}{l}34,4 \\
40,9\end{array}$ & $+0,06$ & 0,38 & $b$ \\
\hline 4 & 8 & $\begin{array}{l}34,5 \\
45,0\end{array}$ & $+0,32$ & 0,70 & $a b$ & 12 & $\begin{array}{l}36,6 \\
44,4\end{array}$ & $+0,03$ & 0,28 & $a b$ & 13 & $\begin{array}{l}33,0 \\
42,9\end{array}$ & $+0,57$ & 0,34 & c \\
\hline $5 \perp$ & 11 & $\begin{array}{l}26,9 \\
53,1\end{array}$ & $-0,29$ & 0,69 & ac & 15 & $\begin{array}{l}35,1 \\
55,3\end{array}$ & $+0,03$ & 0,46 & $a b$ & 14 & $\begin{array}{l}34,3 \\
42,3\end{array}$ & $-0,22$ & 0,47 & $a b$ \\
\hline 6 & 15 & $\begin{array}{l}35,4 \\
49,0\end{array}$ & $+0,30$ & 0,77 & $a b$ & 14 & $\begin{array}{l}35,4 \\
45,1\end{array}$ & $-0,11$ & 0,59 & $a b$ & 15 & $\begin{array}{l}33,5 \\
42,7\end{array}$ & $+0,01$ & 0,45 & $a b$ \\
\hline 7 & 15 & $\begin{array}{l}39,7 \\
59,2\end{array}$ & $-0,69$ & 1,07 & c & 14 & $\begin{array}{l}36,3 \\
47,9\end{array}$ & $-0,20$ & 0,38 & a & & & & & \\
\hline 8 & 15 & $\begin{array}{l}35,6 \\
44,3\end{array}$ & $-0,14$ & 0,76 & $a b c$ & 15 & $\begin{array}{c}35,1 \\
44,1\end{array}$ & $+0,05$ & 0,38 & $a b$ & & & & & \\
\hline Total & 102 & $\begin{array}{l}26,9 \\
59,2\end{array}$ & 0,00 & 0,82 & & 115 & $\begin{array}{l}33,0 \\
55,3\end{array}$ & 0,00 & 0,43 & & 78 & $\begin{array}{l}33,0 \\
42,9\end{array}$ & 0,00 & 0,51 & \\
\hline ANOV & & Sy & $=5,13$ & $\begin{array}{l}\mathrm{Sr}= \\
\mathrm{F}=\end{array}$ & $\begin{array}{l}0,77 \\
3,116(* *)\end{array}$ & & Sy $=$ & 3,35 & $\begin{array}{l}\mathrm{Sr}= \\
\mathrm{F}=1\end{array}$ & $\begin{array}{l}0,43 \\
, 205 \text { (NS) }\end{array}$ & & Sy $=2$ & & $\begin{array}{l}\mathrm{Sr}=0, \\
\mathrm{~F}=6,7\end{array}$ & $\left({ }^{* * *}\right)$ \\
\hline
\end{tabular}


lement de la région Finistère (région 4 ) dont la moyenne de $+0,57 \mathrm{~g} / \mathrm{kg}$ est significativement différente des 5 autres régions (Tableau II).

Mis à part ce département, on distingue deux groupes homogènes, ne différant que par leurs éléments extrêmes, le Doubs et l'Aisnes (régions 1 et 3 ).

L'amplitude maximale des moyennes de l'essai passe de $0,85 \mathrm{~g} / \mathrm{kg}$ à $0,34 \mathrm{~g} / \mathrm{kg}$ lorsqu'on élimine la région Finistère; les écarts types régionaux varient de 0,34 à $0,56 \mathrm{~g} / \mathrm{kg}$, avec une valeur moyenne à $0,44 \mathrm{~g} / \mathrm{kg}$.

\section{Filtre $\mathbf{B}(\mathbf{3 , 4 8} \mu \mathrm{m})$}

Les écarts types résiduels des régressions, mesurant la précision globale observée lors des 3 essais s'élèvent respectivement à $0,52,0,27$ et $0,31 \mathrm{~g} / \mathrm{kg}$ (Tableau III).

\section{Premier essai}

II montre une hétérogénéité régionale hautement significative, provenant de deux régions qui se démarquent nettement des autres $\left(n^{\circ} 7\right.$ et 8 , Aveyron et Monts-duLyonnais).

L'amplitude maximale entre les moyennes de résidus régionales de $0,63 \mathrm{~g} / \mathrm{kg}$ (significatif) passe à $0,19 \mathrm{~g} / \mathrm{kg}$ (non significatif) en l'absence de ces deux régions.

Les écarts types des écarts régionaux fluctuent entre 0,25 et $0,77 \mathrm{~g} / \mathrm{kg}$, avec pour valeur moyenne $0,47 \mathrm{~g} / \mathrm{kg}$.

\section{Deuxième essai}

II ne présente pas d'influence régionale significative, les huit régions comparées for- mant un groupe homogène (zones non distinctes 2 à 2, Tableau III).

L'amplitude maximale observée est de $0,17 \mathrm{~g} / \mathrm{kg}$ (non significative) tandis que les écarts types par région se situent entre 0,17 et $0,38 \mathrm{~g} / \mathrm{kg}$, avec un écart type moyen intrarégion de $0,27 \mathrm{~g} / \mathrm{kg}$.

\section{Troisième essai}

II ne montre pas non plus d'effet régional significatif au seuil de $5 \%$ et, si le département du Doubs (région 1) se démarque significativement de ceux des Deux-Sèvres et de la Mayenne (régions 2 et 6 ), l'écart maximal entre régions ne dépasse pas $0,31 \mathrm{~g} / \mathrm{kg}$ (Tableau III).

L'écart type moyen intrarégion reste faible, $0,31 \mathrm{~g} / \mathrm{kg}$, pour des dispersions régionales semblables à celles du $2^{\mathrm{e}}$ essai $(0,24-0,37 \mathrm{~g} / \mathrm{kg})$.

\section{Les dosages de protéines}

Les valeurs observées pour les écarts types résiduels des régressions varient de 0,18 à $0,29 \mathrm{~g} / \mathrm{kg}$ (Tableau/IV et V). Elles sont faibles dans l'absolu, montrant par la l'excellente précision d'estimation de la méthode (Grappin, 1976).

L'analyse des variances ne met en évidence aucune influence régionale sur la précision des résultats instrumentaux, que les dosages aient bénéficié d'une correction, fonction du taux de lactose, ou non.

L'utilisation d'une intercorrection lactose réduit légèrement les écarts types des résidus régionaux, mais de manière non significative. Ce léger gain permet néanmoins de faire apparaître des différences significatives entre régions, qui sont masquées en l'absence de correction (essais 1 et 3). 
Tableau III. Tableau des moyennes (d) et des écarts types régionaux (Sd) des écarts aux régressions linéaires observés en dosage de matière grasse à $3,48 \mu \mathrm{m}$, sur les 3 essais successifs.

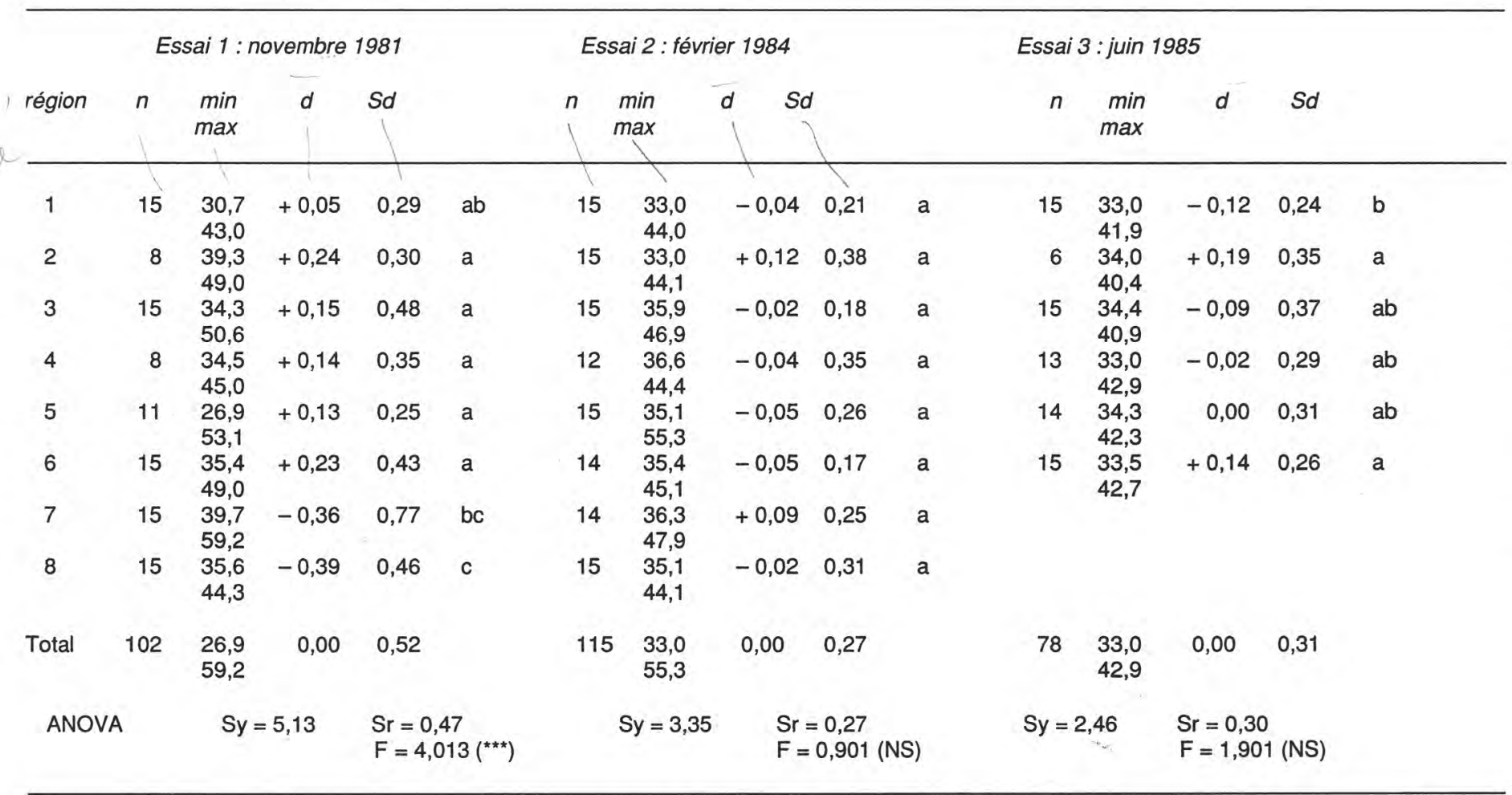

Sy : écart type des taux de référence; $\mathrm{Sr}$ : écart type résiduel (intrarégion) de l'analyse de variance à 1 critère; $F$ : test $F$ sur le facteur région; a, b, $\mathrm{c}:$ moyennes de même lettre non significativement differentes 2 a 2 au seuil de $5 \%$; (NS) : non significatif au seuil de $5 \% ;\left(*^{*}\right):$ significatif $(0,01<P<0,05) ;\left(^{* *}\right):$ hautement significatif $(0.001<P<0.01) ;\left(^{* * *}\right)$ : très hautement significatif $(P<0,001)$. 
Tableau IV. Tableau des moyennes (d) et des écarts types régionaux (Sd) des écarts aux régressions linéaires observés en dosage de protéines à $6,46 \mu \mathrm{m}$ avec intercorrection lactose, sur les 3 essais successifs.

\begin{tabular}{|c|c|c|c|c|c|c|c|c|c|c|c|c|c|c|c|}
\hline \multirow[b]{2}{*}{ région } & \multicolumn{4}{|c|}{ Essai 1 : novembre 1981} & & \multicolumn{4}{|c|}{ Essai 2 : février 1984} & & \multicolumn{3}{|c|}{ Essai 3 : juin 1985} & \multirow[b]{2}{*}{$S d$} & \\
\hline & $n$ & $\begin{array}{l}\min \\
\max \end{array}$ & $d$ & $S d$ & & $n$ & $\min$ & $d$ & \multicolumn{2}{|l|}{$S d$} & $n$ & $\min _{\max }$ & $d$ & & \\
\hline 1 & 15 & $\begin{array}{l}29,3 \\
37,1\end{array}$ & 0,00 & 0,24 & $a b$ & 15 & $\begin{array}{l}26,3 \\
33,4\end{array}$ & $+0,10$ & 0,29 & $a$ & 15 & $\begin{array}{l}29,0 \\
33,7\end{array}$ & $-0,02$ & 0,18 & $a b$ \\
\hline 2 & 8 & $\begin{array}{l}30,7 \\
35,5\end{array}$ & $-0,02$ & 0,19 & $a b$ & 15 & $\begin{array}{l}27,5 \\
37,5\end{array}$ & $+0,07$ & 0,28 & $a$ & 7 & $\begin{array}{l}29,3 \\
31,3\end{array}$ & $+0,10$ & 0,26 & a \\
\hline 3 & 15 & $\begin{array}{l}29,4 \\
35,9\end{array}$ & $+0,03$ & 0,19 & b & 15 & $\begin{array}{l}29,2 \\
32,9\end{array}$ & 0,00 & 0,27 & $a$ & 15 & $\begin{array}{l}28,8 \\
32,9\end{array}$ & $-0,12$ & 0,16 & b \\
\hline 4 & 8 & $\begin{array}{l}32,0 \\
37,9\end{array}$ & $+0,05$ & 0,26 & $a b$ & 12 & $\begin{array}{l}27,8 \\
34,1\end{array}$ & $+0,05$ & 0,33 & $a$ & 13 & $\begin{array}{l}28,7 \\
34,5\end{array}$ & $-0,01$ & 0,12 & $a b$ \\
\hline 5 & 11 & $\begin{array}{l}29,6 \\
35,1\end{array}$ & $-0,13$ & 0,12 & a & 15 & $\begin{array}{l}26,7 \\
33,7\end{array}$ & $-0,06$ & 0,23 & a & 14 & $\begin{array}{l}28,3 \\
31,9\end{array}$ & $+0,06$ & 0,16 & a \\
\hline 6 & 15 & $\begin{array}{l}29,0 \\
36,2\end{array}$ & $+0,04$ & 0,23 & b & 14 & $\begin{array}{l}29,6 \\
34,6\end{array}$ & $-0,07$ & 0,31 & a & 15 & $\begin{array}{l}27,7 \\
33,5\end{array}$ & $+0,05$ & 0,21 & a \\
\hline 7 & 15 & $\begin{array}{l}30,4 \\
37,8\end{array}$ & $+0,03$ & 0,31 & $a b$ & 14 & $\begin{array}{l}28,1 \\
37,8\end{array}$ & $-0,09$ & 0,25 & a & & & & & \\
\hline 8 & 15 & $\begin{array}{l}29,8 \\
34,6\end{array}$ & $-0,02$ & 0,24 & $a b$ & 15 & $\begin{array}{l}28,1 \\
31,4\end{array}$ & $+0,01$ & 0,31 & a & & & & & \\
\hline Total & 102 & $\begin{array}{l}29,0 \\
37,8\end{array}$ & 0,00 & 0,23 & & 115 & $\begin{array}{l}26,3 \\
37,8\end{array}$ & 0,00 & 0,28 & & 79 & $\begin{array}{l}27,7 \\
34,5\end{array}$ & 0,00 & 0,19 & \\
\hline ANOV & & Sy & $=2,08$ & $\begin{array}{l}\mathrm{Sr}=0 \\
\mathrm{~F}=0\end{array}$ & 40 (NS) & & Sy $=1$ & & $\begin{array}{l}\mathrm{Sr}=0,2 \\
\mathrm{~F}=0,85\end{array}$ & (NS) & Sy & $=1,58$ & $\begin{array}{l}\mathrm{Sr}= \\
\mathrm{F}=\end{array}$ & $\begin{array}{l}18 \\
342 \text { (NS) }\end{array}$ & \\
\hline
\end{tabular}


Tableau V. Tableau des moyennes (d) et des écarts types régionaux (Sd) des écarts aux régressions linéaires observés en dosage de protéines à 6,46 $\mu \mathrm{m}$ sans intercorrection lactose, sur les 3 essais successifs.

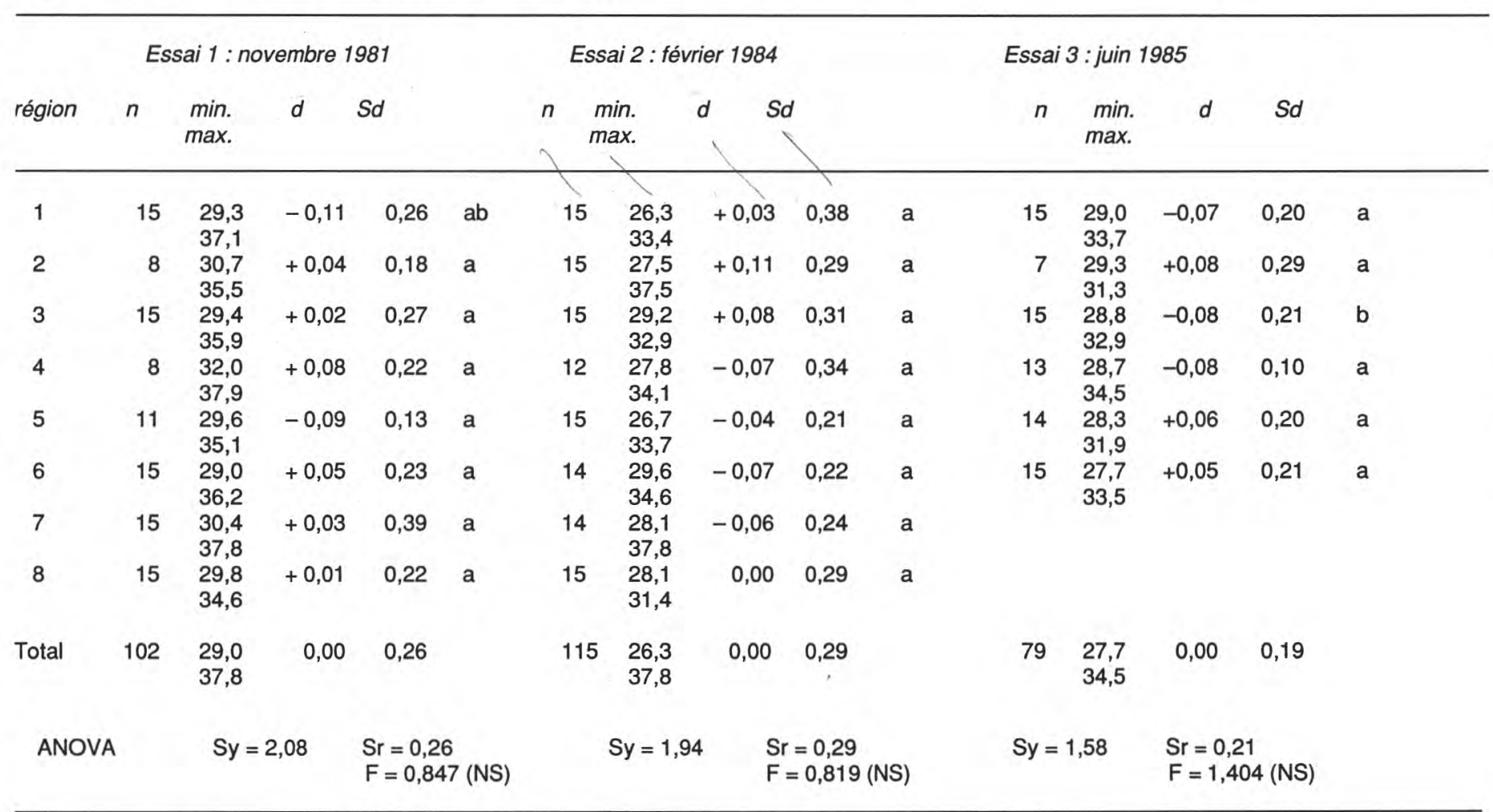

Sy : écart type des taux de référence; $\mathrm{Sr}$ : écart type résiduel (intrarégion) de l'analyse de variance à 1 critère; $\mathrm{F}$ : test $\mathrm{F}$ sur le facteur région; a, b, $\mathrm{c}:$ moyennes de même lettre non significativement différentes 2 à 2 au seuil de $5 \%$; (NS) : non significatif au seuil de $5 \%$; $\left(^{*}\right)$ : significatif $(0,01<P<0,05)$; $\left({ }^{* *}\right)$ : hautement significatif $(0,001<P<0,01) ;\left({ }^{* * *}\right)$ : très hautement significatif $\quad(P<0,001)$. 
Les amplitudes maximales entre régions sont, respectivement pour les essais successifs, avec la correction lactose de 0,18 (significatif), 0,19 (non significatif) et $0,22 \mathrm{~g} / \mathrm{kg}$ (significatif), et sans la correction lactose de $0,19,0,18$ et $0,16 \mathrm{~g} / \mathrm{kg}$ (non significatif).

\section{DISCUSSION}

\section{Hétérogénéité régionale et étalonnage en matière grasse}

Les écarts régionaux sont marqués principalement en période de transition alimentaire, ce qu'illustrent les résultats de l'essai 1 (novembre 81).

En effet, selon la situation géographique régionale, les animaux sont à un stade plus ou moins avancé du changement de régime alimentaire été-hiver entraînant un étalement des écarts entre régions; de même au sein des régions, la transition n'est pas simultanée pour l'ensemble des élevages, aussi observe-t-on également un étalement des dispersions entre troupeaux d'une même région.

L'influence des régions, dans cet essai, touche essentiellement, les dosages de matière grasse, et plus particulièrement les analyses avec filtre $A$, même si les différences régionales en filtre $B$ apparaîssent plus significatives.

La valeur de précision globale de 0,82 $\mathrm{g} / \mathrm{kg}$ observée en filtre A $(5,73 \mu \mathrm{m})$ est audelà de la valeur acceptable en matière de précision à un niveau départemental. $\mathrm{Ce}$ n'est, par contre, pas le cas pour la valeur observée en filtre $B(3,48 \mu \mathrm{m})$, de $0,52 \mathrm{~g} /$ $\mathrm{kg}$, en principe admissible d'après les limites fixées dans la norme ITEB-FNOCL (1983).

L'essai $n^{\circ} 2$ (février 84 ) s'est déroulé en une période de stabilité alimentaire, les réserves fourragères n'étant, en principe, pas épuisées. Cela concorde avec les faibles valeurs d'écarts types résiduels observées, tant en filtre $A$ qu'en filtre $B$. Celles-ci sont du même ordre de grandeur que ce qui est généralement observé à l'occasion d'un étalonnage départemental.

L'absence d'effet régional et l'étroitesse de la précision d'estimation de la méthode constatées à cette période autorisent sans restriction la mise en place d'un calibrage unique pour l'ensemble des régions présentes.

L'essai $n^{\circ} 3$ (juin 85), également effectué à une période de stabilité de régime alimentaire présente, de la même manière, de faibles écarts types par région, indicateurs de pratiques alimentaires homogènes région par région.

Néanmoins, la présence d'une région (Finistère) nettement démarquée des autres, en analyse avec filtre A, montre que des particularités locales en matière d'affouragement ou de conduite du troupeau peuvent amener des biais régionaux non négligeables qui vont à l'encontre des perspectives d'un étalonnage unique centralisé.

Si la valeur de précision globale de 0,51 $\mathrm{g} / \mathrm{kg}$ est acceptable, il peut être hasardeux, vu l'amplitude régionale de $0,85 \mathrm{~g} / \mathrm{kg}$ occasionnée par la région Finistère et le petit nombre de régions testées dans l'essai, de se satisfaire d'un calibrage unique, en filtre $A$.

L'absence d'effet régional et les faibles valeurs de précisions obtenues avec le filtre B, par contre, le permettent aisément. 


\section{Hétérogénéité régionale et étalonnage en protéines}

Les 3 essais témoignent d'une variabilité régionale quasi inexistante sur les périodes et les régions étudiées.

Les amplitudes maximales entre régions, qu'elles soient significatives ou non, ont été dans tous les cas inférieures ou très proches de $0,20 \mathrm{~g} / \mathrm{kg}$. Ces valeurs très faibles montrent que l'écart systématique à la méthode de référence des régions les plus extrêmes est quasi négligeable dans le cadre d'un étalonnage commun en regard des autres sources de biais analytiques.

Les écarts types résiduels des régressions, tous inférieurs à $0,30 \mathrm{~g} / \mathrm{kg}$, indiquent que les facteurs d'erreur liée à la méthode ont été d'une faible incidence dans le cadre de ces essais. Ils indiquent, en d'autres termes, que les teneurs en lactose et citrates étaient d'une variabilité limitée dans les laits de tanks prélevés au cours des essais.

Compte tenu de la qualité de la précision globale obtenue, tout porte à croire qu'un calibrage unique pour les régions testées est possible.

\section{Comparaison filtre $\boldsymbol{A}(5,73 \mu \mathrm{m})$ / filtre $\boldsymbol{B}$ $(3,48 \mu \mathrm{m})$}

La comparaison des deux types de dosages de la matière grasse fait apparaître une nette supériorité du filtre $B$, en ce qui concerne la précision des dosages.

Le gain de précision mesuré sur les écarts types résiduels des régressions des essais est en moyenne de $38 \%$. Cette amélioration apparaît, aussi bien au niveau de l'amplitude des écarts moyens régionaux, qu'au niveau des écarts types in- trarégionaux, l'utilisation du filtre B réduisant, dans la grande majorité des cas, les écarts moyens régionaux (Tableaux II et III).

\section{CONCLUSION}

D'après les résultats des 3 essais, il apparaît que le système d'étalonnage à préconiser sur un ensemble de régions tel que celui étudié, dépend essentiellement du choix du filtre utilisé pour le dosage de la matière grasse par l'ensemble des laboratoires.

Pour le dosage de la matière grasse, les laboratoires de contrôle laitier et de paiement du lait utilisent pour la plupart le filtre A $(5,73 \mu \mathrm{m})$. De ce fait, compte tenu de l'importance des écarts régionaux observés, un système d'étalonnage individuel propre à chaque laboratoire apparaît le mieux adapté pour s'affranchir des écarts systématiques dus à la composition de la matière grasse et répondre aux objectifs de précision souhaités.

En effet les 2 périodes critiques de changement de régime alimentaire (automne et printemps) bien connues des laboratoires, peuvent rendre l'adoption d'un étalonnage unique centralisé hasardeuse, d'autant qu'il n'est pas garanti qu'en d'autres périodes, des hétérogénéités régionales n'apparaissent pas, pour des raisons naturelles particulières.

L'utilisation d'échantillons de référence communs à tous les laboratoires ne pourrait être qu'une solution partielle à l'étalonnage des appareils. Elle permettrait de vérifier la linéarité des appareils et d'effectuer un premier ajustement de pente, lequel devrait être affiné localement à l'aide d'un échantillon représentatif de la population de laits de la zone de collecte. 
Dans cet esprit, il peut être envisagé de faire évaluer périodiquement par le laboratoire pilote les écarts systématiques propres à chaque laboratoire. Celui-ci pourrait, soit fournir les valeurs de réglage optimales permettant un ajustement plus fin, soit donner pour les échantillons standards, directement des taux de référence prenant en compte les différences locales à corriger, solution similaire à ce qui est fait en Suède sur 18 laboratoires équipés en filtre $A$.

Dans le cas d'une utilisation généralisée du filtre $B$, un étalonnage unique peut être envisagée sans restriction. En effet, les hétérogénéités régionales ponctuelles apparaissent suffisamment faibles pour que le bilan global de qualité apporté par un tel système soit positif (suppression de l'erreur sur les méthodes de référence, facilité de calibrage dans les laboratoires). C'est notamment le choix qui a été fait au Royaume-Uni où, depuis plusieurs années déjà, des échantillons étalons sont adressés régulièrement à 7 laboratoires régionaux équipés en filtre $B(3,48 \mu \mathrm{m})$.

Les observations faites sur les dosages de protéines montrent d'une part l'absence de tout effet régional et, d'autre part, une très bonne précision de la méthode observée lors de chaque essai. De même que pour les dosages avec filtre B l'étalonnage unique centralisé apparaît comme une possibilité offerte aux laboratoires.

\section{RÉFÉRENCES}

Brochet M. (1982) Etude des laits de brebis Méthodes de dosage des principaux constitutants et composition des protéines. Thèse de $3^{e}$ cycle. $n^{\circ} 1 / 190$. Université Claude Bernard, Lyon I
Gaudilliêre T. \& Grappin R. (1982) Influence de l'acide citrique sur le dosage des protéines du lait par la méthode infrarouge. Mémoire de fin d'études, SPILAB, Douai

Grappin R. (1976) Guide pour l'évaluation des méthodes d'analyses de routine. Lait 56,608 621

Grappin R. \& Jeunet R. (1972) Facteurs biologiques responsables des variations observées dans la précision des méthodes de routine utilisées pour le dosage de la matière grasse du lait (Milkotester, Infra-Red Milk Analyser et Gerber). Lait 52, 324-346

Grappin R. \& Jeunet R. (1976) Essais de l'appareil Milkoscan 300 utilisé pour le dosage en série de la matière grasse et des protéines du lait. Lait 56, 498-520

Grappin R. \& Jeunet (1979) Méthodes de routine pour le dosage de la matière grasse et des protéines du lait de chèvre. Lait 59, 345-360

Grappin R. \& Jeunet R. (1981) Influence of lipol$y$ sis on gravimetric and routine methods for milk fat test. 76th Annual Meeting of the American Dairy Science Association, Louisiana

ITEB-FNOCL (1983) Norme d'utilisation des appareils de dosage par absorption infra rouge utilisés pour la mesure des teneurs en matière grasse, protéines et lactose du lait. ITEB, 149 rue de Bercy, 75012 Paris

Kerkhof Mogot M.F., Koops J., Neeter R., Slangen K.J., Van Hemert H., Kooyman O. \& Wooldrix $H$. (1982) Routine testing of farm tank milk with the MilkoScan 203. 1) Calibration procedure and small scale experiments. Neth. Milk Dairy J. 36, 115-133

Leray O. (1984) Méthodes Instrumentales de Dosage des Constituants du lait. Colloque INRA-ENSAR-INAPG, Rennes

Shields J. (1975) The analysis of milk by infrared absorption. B. Ph. Thesis. Chemistry Department. University of York, York, UK

Sjaunja L.O. (1982) Studies on milk analysis of individual cow milk samples. Report $n^{\circ} 56$. Department of Animal Breeding and Genetics

Sjaunja L.O. \& Anderson I. (1985) Laboratory experiments with a nex infra-red (IR) milk analyser, the Milko-Scan 605. Acta Agric. Scand. 35, 345-352

Swedish University of Agricultural Sciences. Uppsala, Suède 